LBNL- 42525

UCB-PTH-98/55

hep-ph/98

\title{
Leading Order Textures for Lepton Mass Matrices ["
}

\author{
Lawrence J. Hall and David Smith \\ Department of Physics and \\ Theoretical Physics Group, Lawrence Berkeley National Laboratory \\ University of California, Berkeley, California 94720
}

\begin{abstract}
In theories with three light neutrinos, certain simplicity assumptions allow the construction of a complete list of leading order lepton mass matrices. These matrices are consistent with $m_{\tau} \neq 0, \Delta m_{12}^{2} \ll \Delta m_{23}^{2}, \theta_{23} \sim O(1)$ and $\theta_{13}=0$, as suggested by measurements of atmospheric and solar neutrino fluxes. The list contains twelve generic cases: two have three degenerate neutrinos, eight have two neutrinos forming a Dirac state, and in only two cases is one neutrino much heavier than the other two. For each of these twelve generic cases the possible forms for the perturbations which yield $m_{\mu}$ are given. Ten special textures are also found.
\end{abstract}

${ }^{*}$ This work was supported in part by the U.S. Department of Energy under Contracts DE-AC0376SF00098, in part by the National Science Foundation under grant PHY-95-14797. 


\section{Introduction}

Over the last several decades, experiments have revealed a striking generational pattern of quark and charged lepton masses and mixings. In each charged sector there is a strong hierarchy of mass eigenvalues between the three generations, $m_{3} \gg m_{2} \gg m_{1}$, and the three angles describing mixing between the left-handed charge $2 / 3$ quarks and charge $-1 / 3$ quarks are all small. The origin of this pattern, and of the precise values of the flavor observables, has been greatly debated, with several diverse approaches and very many competing theories.

Despite this diversity, a common theme can be identified: the fermion masses are to be understood in an expansion, in which the leading order term for each charged sector has the form

$$
m^{(0)}=\left(\begin{array}{lll}
0 & 0 & 0 \\
0 & 0 & 0 \\
0 & 0 & A
\end{array}\right) .
$$

This gives the leading order results $m_{3} \gg m_{2}=m_{1}=0$, and vanishing mixing angles to the third generation. Indeed, for the charged sectors it has seemed self evident that this $i s$ the leading order structure, and the debate has centered on higher order terms in the expansion.

The Super-Kamiokande measurements of the atmospheric neutrino fluxes [1] cast considerable doubt on (回) as the correct leading order form, at least in the lepton sector. These measurements are of great importance not just because they provide strong evidence for neutrino masses: they may also fundamentally change our view of the pattern of flavor symmetry breaking.

The interpretation of this atmospheric $\nu$ flux data in terms of neutrino oscillations implies a large mixing angle $\left(\theta>32^{\circ}\right)$ between $\nu_{\mu}$ and some other neutrino state, which could have large $\nu_{\tau}$ or singlet neutrino components, but only a small $\nu_{e}$ component. Is it possible to reconcile this observation with the leading order form (近)? This issue is especially important in unified theories, where relations are expected between the textures for the various charged sectors. We are aware of three possible resolutions, each of which can be criticized:

- In a three generation theory, with each generation containing a right-handed neutrino, it is possible to write down textures for charged leptons, $\left(m_{E}\right)$, Dirac neutrino masses, $\left(m_{L R}\right)$, and right-handed Majorana masses, $\left(m_{R R}\right)$, which all reduce to (11) at leading order, but which give a leading order form to $m_{L L}=m_{L R} m_{R R}^{-1} m_{L R}^{T}$ which is very different from (1), and has dominant terms giving $\theta_{\mu \tau} \approx O(1)$. However, for this to happen the 23 and 33 entries of $m_{L L}$ need to be comparable, and since they arise from different terms in $m_{L R}$ and $m_{R R}$ the large value for $\theta_{\mu \tau}$ appears to be accidental. 
- In a three generation theory, even if both $m_{E}$ and $m_{L L}$ have the leading order form of (1), the 23 entries may not be much smaller than the 33 entries, generating a significant $\theta_{\mu \tau}$ [2]. For example, in this conventional hierarchical scheme, the ratios of eigenvalues in the charged and neutral sectors suggest charged and neutral contributions to $\theta_{\mu \tau}$ of about $14^{\circ}$ and $18^{\circ}$ respectively. Providing the relative sign is such that these contributions add, large $\mu-\tau$ mixing can result. This is an important observation, because it shows that the conventional picture, where all textures have the leading order form of (11), is not excluded by the Super-Kamiokande data. However, the data does prefer an even bigger angle: the conventional picture is now disfavored.円

- In a theory with more than three light neutrino states it may be that (1) gives the correct leading order neutrino masses terms between the 3 left-handed states, but there is some additional mass term coupling $\nu_{\mu}$ to a light singlet state leading to large mixing between these states [3]. Such schemes are certainly non-minimal, and must answer three questions. Why is there a singlet state? Why is it so light? Why is it coupled to $\nu_{\mu}$ rather than to $\nu_{\tau}$ or $\nu_{e}$ ? Furthermore, during big bang nucleosynthesis the fourth state is kept in thermal equillibrium by oscillations, and the resulting extra contribution to the energy density is disfavored by observations which allow the primordial abundances of D and ${ }^{4} \mathrm{He}$ to be inferred [5].

In view of these criticisms of the conventional leading order texture (1), in this paper we study an alternative, straightforward and direct interpretation of the data: in a three generation theory large $\theta_{\mu \tau}$ arises because $m_{L L}$ and/or $m_{E}$ have a leading order form which differs from (11). In the bulk of this paper, we perform an analysis to find all possible leading order textures for $\left(m_{E}, m_{L L}\right)$, subject to a simplicity assumption, such that there is a hierarchy of neutrino mass splittings: $\Delta m_{23}^{2} \gg \Delta m_{12}^{2}$ as prefered by atmospheric and solar neutrino data.2

\footnotetext{
${ }^{1}$ One might try to argue that the conventional picture could even give $45^{\circ}$ mixing if the hierarchy between the two $\Delta m^{2}$ is reduced. This is permissable if one of the solar neutrino experiments, or the standard solar model, is incorrect [4]. However, in this case the 23 entry in $m_{L L}$ is no longer small enough to be considered subleading.

${ }^{2}$ In [6] a texture analysis is done to find the possible leading-order forms for $m_{L L}$, in the chargediagonal basis, that have either maximal mixing for $\theta_{23}$ alone or maximal mixing for both $\theta_{23}$ and $\theta_{12}$.
} 


\section{Texture Analysis: Rules}

In theories with three light neutrinos, the leading order, real, diagonal mass matrices consistent with $\Delta m_{12}^{2} \ll \Delta m_{23}^{2}$ are

$$
\bar{m}_{L L}^{I}=\left(\begin{array}{ccc}
0 & 0 & 0 \\
0 & 0 & 0 \\
0 & 0 & \alpha
\end{array}\right) \quad \bar{m}_{L L}^{I I}=\left(\begin{array}{ccc}
\alpha & 0 & 0 \\
0 & \alpha & 0 \\
0 & 0 & 0
\end{array}\right) \quad \bar{m}_{L L}^{I I I}=\left(\begin{array}{ccc}
\alpha & 0 & 0 \\
0 & \alpha & 0 \\
0 & 0 & \beta
\end{array}\right) \quad \bar{m}_{L L}^{I V}=\left(\begin{array}{ccc}
\alpha & 0 & 0 \\
0 & \alpha & 0 \\
0 & 0 & \alpha
\end{array}\right)
$$

for the neutrinos, and

$$
\bar{m}_{E}=\left(\begin{array}{ccc}
0 & 0 & 0 \\
0 & 0 & 0 \\
0 & 0 & \gamma
\end{array}\right)
$$

for the charged leptons. In $\bar{m}_{L L}^{I I I}, \alpha$ and $\beta$ are of the same order but not equal. The diagonal mass matrices are related to $m_{L L}$ and $m_{E}$, the mass matrices in the flavor basis, by unitary transformations:

$$
m_{L L}=V_{\nu}^{*} \bar{m}_{L L} V_{\nu}^{\dagger} \quad m_{E}=V_{E_{L}} \bar{m}_{E} V_{E_{R}}^{\dagger}
$$

The leptonic mixing matrix $V=V_{E_{L}}^{\dagger} V_{\nu}$ then relates the neutrino weak and mass eigenstates according to

$$
\nu_{e_{i}}=V_{i j} \nu_{j}
$$

and can be parametrized by

$$
V=R_{23}\left(\theta_{23}\right) R_{13}\left(\theta_{13}\right)\left(\begin{array}{ccc}
1 & 0 & 0 \\
0 & e^{i \beta} & 0 \\
0 & 0 & 1
\end{array}\right) R_{12}\left(\theta_{12}\right)\left(\begin{array}{ccc}
1 & 0 & 0 \\
0 & e^{i \alpha_{1}} & 0 \\
0 & 0 & e^{i \alpha_{2}}
\end{array}\right)
$$

If $\Delta m_{23}^{2}>2 \cdot 10^{-3} \mathrm{eV}^{2}$, then results from the Chooz experiment require $\theta_{13}<13^{\circ}$ ]. In fact, even if $\Delta m_{23}^{2}<2 \cdot 10^{-3} \mathrm{eV}^{2}$, fits to the Super-Kamiokande atmospheric data (for $\left.\Delta m_{12}^{2} \ll \Delta m_{23}^{2}\right)$ alone restrict $\theta_{13}<20^{\circ}$ [8]. In light of these constraints we will assume that the leading order contribution to $\theta_{13}$ vanishes, giving

$$
V=R_{23}\left(\theta_{23}\right) R_{12}\left(\theta_{12}\right)\left(\begin{array}{ccc}
1 & 0 & 0 \\
0 & e^{i \alpha_{1}} & 0 \\
0 & 0 & e^{i \alpha_{2}}
\end{array}\right)
$$

with $\theta_{23}$ of order unity, as suggested by Super-Kamiokande results.

Our aim is to perform a systematic search for leading order leptonic mass matrices $m_{L L}$ and $m_{E}$ that have the following features:

- Diagonalizing them gives $\bar{m}_{E}$ of (3) for the charged leptons and one of the $\bar{m}_{L L}$ 's of (2) for the neutrinos. 
- They produce a leptonic mixing matrix that can be paramatrized as in (7), with $\theta_{23} \sim 1$.

- Their forms offer the hope of a simple explanation in terms of flavor symmetries.

Because we are particularly interested in leading order $m_{L L}$ and $m_{E}$ that can be simply understood using flavor symmetries, we constrain their forms by allowing only the following exact relations between non-vanishing elements:

- They may be equal up to a phase.

- They may be related so as to give a vanishing determinant or sub-determinant.

The latter class of relations is allowed because, as discussed in [4, 9, 10], vanishing determinants arise naturally when heavy particles are integrated out, as in the seesaw mechanism.

As an illustration of how these rules are used, consider applying a (2-3) rotation, first on $\bar{m}_{L L}^{I}$, and second on $\bar{m}_{L L}^{I I}$. In the first case we get a neutrino mass matrix of the form

$$
m_{L L}=\left(\begin{array}{ccc}
0 & 0 & 0 \\
0 & \frac{B^{2}}{A} & B \\
0 & B & A
\end{array}\right)
$$

Our rules allow this matrix because the relation among elements yields a vanishing subdeterminant. In the second case the transformation gives

$$
m_{L L}=\left(\begin{array}{ccc}
\frac{B^{2}}{A}+A & 0 & 0 \\
0 & \frac{B^{2}}{A} & B \\
0 & B & A
\end{array}\right)
$$

(ignoring possible phases). This matrix is not allowed because the relation between the 11, 22, and 33 entries is not essential for the vanishing of any determinant. Cases such as (9) are not excluded because it is impossible to attain them from a theory with a flavor symmetry. Rather, they are excluded for reasons of simplicity: in our judgement it is more difficult to construct such theories, compared with theories for textures with all non-zero entries independent, equal up to a phase, or related to give a vanishing determinant.

Given a pairing of leading order $\left(m_{L L}, m_{E}\right)$ that satisfies our simplicity requirement, and that has mass eignenvalues consistent with (2) and (3), it is straightforward to determine whether or not $\theta_{23} \sim 1$ is satisfied. Unfortunately, the remaining requirement, $\theta_{13} \sim 0$, is rendered meaningless by the leading order relation $m_{e}=m_{\mu}=0$. This is easily seen by rotating the left-handed doublets to diagonalize $m_{L L}$, and then rotating the right-handed charged leptons to give

$$
m_{E}=\left(\begin{array}{lll}
0 & 0 & 0 \\
0 & 0 & 0 \\
0 & 0 & A
\end{array}\right),\left(\begin{array}{lll}
0 & 0 & 0 \\
0 & 0 & B \\
0 & 0 & A
\end{array}\right), \text { or }\left(\begin{array}{lll}
0 & 0 & C \\
0 & 0 & B \\
0 & 0 & A
\end{array}\right)+\text { perturbations. }
$$


We can diagonalize each leading order piece in (10) by applying (at most) a diagonal phase rotation followed by (1-2) and (2-3) rotations. This indicates that, if we ignore the perturbations responsible for the muon mass, we are free to choose $\theta_{13}=0$ for any leading order $\left(m_{L L}, m_{E}\right)$ pairing.

Although it is impossible to use the $\theta_{13} \sim 0$ requirement to restrict lepton mass matrices based on leading order considerations alone, it is true that it is easier for some $\left(m_{L L}, m_{E}\right)$ pairings than it is for others to add perturbations that give $\theta_{13} \sim 0$. As we will see, some pairings require special relations among the perturbations that seem difficult to understand by symmetry considerations. To exclude these cases we impose a final requirement on our leading order $\left(m_{L L}, m_{E}\right)$ pairings:

- It must be possible to add to $m_{L L}$ and $m_{E}$ perturbations that establish $\theta_{13} \sim 0$ and that satisfy the same simplicity requirements already imposed on the leading order entries: non-vanishing perturbations must be either independent, equal up to a phase, or related in a way that gives a vanishing determinant. We require that the perturbations in $m_{E}$ give $m_{\mu} \neq 0$ while preserving $m_{e}=0$. For the case of three nearly degenerate neutrinos we require that the perturbations in $m_{L L}$ establish $\Delta m_{12}^{2} \ll \Delta m_{23}^{2}$, and for the remaining cases, where $\Delta m_{23}^{2} \neq 0$ is established at leading order, we require that the perturbations in $m_{L L}$ lift the degeneracy between $\nu_{1}$ and $\nu_{2}$.

A simple example will clarify our motives for adding this requirement. Starting with the leading order textures

$$
m_{L L}=\left(\begin{array}{ccc}
0 & 0 & 0 \\
0 & \frac{B^{2}}{A} & B \\
0 & B & A
\end{array}\right) \quad m_{E}=\left(\begin{array}{ccc}
0 & 0 & 0 \\
0 & 0 & E \\
0 & 0 & D
\end{array}\right),
$$

it is easy to find perturbations that satisfy our criteria. For instance, if we add them according to

$$
m_{L L}=\left(\begin{array}{ccc}
0 & 0 & 0 \\
0 & \frac{B^{2}}{A} & B \\
0 & B & A+\epsilon_{2}
\end{array}\right) \quad m_{E}=\left(\begin{array}{ccc}
0 & 0 & 0 \\
0 & 0 & E \\
0 & \epsilon_{1} & D
\end{array}\right),
$$

then in the basis where $m_{L L}$ is diagonal we have

$$
m_{E}=\left(\begin{array}{ccc}
0 & 0 & 0 \\
0 & \epsilon_{1}^{\prime} & E^{\prime} \\
0 & \epsilon_{2}^{\prime} & D^{\prime}
\end{array}\right)
$$

so that $\theta_{13} \sim 0$ is indeed satisfied, and the leading order matrices of (11) are allowed. Things do not work as simply if we instead begin with the leading order pair

$$
m_{L L}=\left(\begin{array}{ccc}
0 & 0 & 0 \\
0 & \frac{B^{2}}{A} & B \\
0 & B & A
\end{array}\right) \quad m_{E}=\left(\begin{array}{ccc}
0 & 0 & F \\
0 & 0 & E \\
0 & 0 & D
\end{array}\right) .
$$


After rotating the lepton doublets to diagonalize $m_{L L}$ we need the form of $m_{E}$ (including perturbations responsible for the muon mass) to have a perturbation only in the 32 entry:

$$
m_{E}=\left(\begin{array}{ccc}
0 & 0 & F \\
0 & 0 & E^{\prime} \\
0 & \epsilon & D^{\prime}
\end{array}\right)
$$

Otherwise, after performing (1-2) and (2-3) rotations to diagonalize the leading order piece of $m_{E}$, we are still left with an additional large (1-2) rotation required to diagonalize the perturbations, which induces a large $\theta_{13}$. One must therefore require that, in the flavor basis, the perturbations enter the charged lepton mass matrix as in

$$
m_{E}=\left(\begin{array}{ccc}
0 & 0 & F \\
0 & \epsilon B & E \\
0 & \epsilon A & D
\end{array}\right)
$$

where $A$ and $B$ are the masses that appear in $m_{L L}$. The non-trivial exact relation required between the perturbations in $m_{E}$ and the leading order entries in $m_{L L}$ indicates that, for generic $A$ and $B$, the textures in (14) do not fulfill our criteria for leading order $\left(m_{L L}, m_{E}\right)$. Note, however, that for the special case $A=B$, the perturbations in (16) are equal, so that the leading order pairing

$$
m_{L L}=\left(\begin{array}{ccc}
0 & 0 & 0 \\
0 & A & A \\
0 & A & A
\end{array}\right) \quad m_{E}=\left(\begin{array}{ccc}
0 & 0 & F \\
0 & 0 & E \\
0 & 0 & D
\end{array}\right)
$$

is allowed by our rules.

\section{Texture Analysis: Results}

The program of our analysis is as follows. First, for each diagonal neutrino and charged lepton mass matrix of (2) and (3), we write down all possible forms for leading order $m_{L L}$ and $m_{E}$ in the flavor basis, consistent with our simplicity requirement restricting relations between non-vanishing elements. For each leading order $\left(m_{L L}, m_{E}\right)$ pairing obtained in this way, we then determine whether there are perturbations that satisfy the criteria described in the preceding paragraphs.

For example, for the case $\bar{m}_{L L}=\bar{m}_{L L}^{I}$ of equation (21), the possible forms for $m_{L L}$ in the flavor basis are

$$
m_{L L}=\left(\begin{array}{ccc}
0 & 0 & 0 \\
0 & 0 & 0 \\
0 & 0 & A
\end{array}\right), \quad m_{L L}=\left(\begin{array}{ccc}
0 & 0 & 0 \\
0 & \frac{B^{2}}{A} & B \\
0 & B & A
\end{array}\right), \text { and } m_{L L}=\left(\begin{array}{ccc}
\frac{C^{2}}{A} & \frac{B C}{A} & C \\
\frac{B C}{A} & \frac{B^{2}}{A} & B \\
C & B & A
\end{array}\right),
$$


and all matrices obtained from these by permuting flavor basis indices. Note that each relation among elements in these matrices leads to a vanishing sub-determinant, and is thus allowed. These forms for $m_{L L}$ may be paired with either

$$
m_{E}=\left(\begin{array}{ccc}
0 & 0 & 0 \\
0 & 0 & 0 \\
0 & 0 & D
\end{array}\right), \quad m_{E}=\left(\begin{array}{ccc}
0 & 0 & 0 \\
0 & 0 & E \\
0 & 0 & D
\end{array}\right), \quad \text { or } m_{E}=\left(\begin{array}{ccc}
0 & 0 & F \\
0 & 0 & E \\
0 & 0 & D
\end{array}\right),
$$

where only the left-handed charged leptons, and not necessarily the right-handed charged leptons, are in their flavor basis. Some $\left(m_{L L}, m_{E}\right)$ pairings from (18) and (19) are immediately excluded because they do not give $\theta_{23} \sim 1$,

$$
m_{L L}=\left(\begin{array}{ccc}
0 & 0 & 0 \\
0 & 0 & 0 \\
0 & 0 & A
\end{array}\right) \quad m_{E}=\left(\begin{array}{ccc}
0 & 0 & 0 \\
0 & 0 & 0 \\
0 & 0 & D
\end{array}\right)
$$

being an obvious example. Other pairings, like that of (14) for generic $A$ and $B$, are excluded because it not possible to add perturbtions that satisfy our requirements. Some pairings that $d o$ work require exact relations between perturbations, as do the leading order textures in (17), while other pairings, such as the one in (11), can accept independent perturbations.

Performing our analysis for each $\bar{m}_{L L}$ of equation (2) leads to the pairings listed in Tables 1-4. Tables 1 and 2 list leading order $\left(m_{L L}, m_{E}\right)$ pairings that can take perturbations with independent magnitudes; these twelve textures we call "generic." Tables 3 and 4 contain pairings that instead require exact relations among perturbations, giving a further ten "special" textures. In Tables 1 and 2 we write the possible forms for $m_{E}$ ast

$$
I \equiv\left(\begin{array}{ccc}
0 & 0 & \epsilon_{3} \\
0 & \epsilon_{1} & \epsilon_{2} \\
0 & 0 & D
\end{array}\right), \quad I I \equiv\left(\begin{array}{ccc}
0 & 0 & \epsilon_{2} \\
0 & \epsilon_{1} & E \\
0 & 0 & D
\end{array}\right), \quad \text { and } I I I \equiv\left(\begin{array}{ccc}
0 & 0 & F \\
0 & 0 & E \\
0 & \epsilon_{1} & D
\end{array}\right)
$$

In Tables 3 and 4 , we instead write $m_{E}$ explicitly and provide an example, for each pairing, of how perturbations can be added to give acceptable masses and mixings. Because for $m_{L L}$ there is often considerable freedom in how perturbations can be added, we show only leading order elements of $m_{L L}$, unless exact relations among these perturbations are required (as they are in the pairings with degenerate neutrinos in Table 3).

\footnotetext{
${ }^{3}$ Because we consider forms for $m_{L L}$ obtained from those in 18 ) by permuting flavor basis indices, there is no need to do the same for $m_{E}$. For example, we consider $m_{L L}=\left(\begin{array}{ccc}\frac{B^{2}}{A} & 0 & B \\ 0 & 0 & 0 \\ B & 0 & A\end{array}\right)$, but not $m_{E}=\left(\begin{array}{ccc}0 & 0 & B \\ 0 & 0 & 0 \\ 0 & 0 & A\end{array}\right)$.

${ }^{4}$ More precisely, the various possible forms for $m_{E}$ can each be brought into one of these three forms by appropriate rotations of the right-handed charged leptons. The perturbations are taken to have comparable magnitudes, but in each matrix only $\epsilon_{1}$ need be non-zero.
} 


\begin{tabular}{|c|c|c|c|}
\hline \hline & $m_{L L}$ & $m_{E}$ & $\bar{m}_{L L}$ \\
\hline 1$)$ & $\left(\begin{array}{ccc}0 & 0 & 0 \\
0 & 0 & 0 \\
0 & 0 & A\end{array}\right)$ & $I I(\mathrm{U}), I I I(\mathrm{LA})$ & $\bar{m}_{L L}^{I}$ \\
$2)$ & $\left(\begin{array}{ccc}0 & 0 & 0 \\
0 & \frac{B^{2}}{A} & B \\
0 & B & A\end{array}\right)$ & $I(\mathrm{U}), I I(\mathrm{U})$ & $\bar{m}_{L L}^{I}$ \\
\hline \hline 3$)$ & $\left(\begin{array}{ccc}A & 0 & 0 \\
0 & A & 0 \\
0 & 0 & A\end{array}\right)$ & $I I(\mathrm{U}), I I I(\mathrm{LA})$ & $\bar{m}_{L L}^{I V}$ \\
$4)$ & $\left(\begin{array}{ccc}0 & A & 0 \\
A & 0 & 0 \\
0 & 0 & A\end{array}\right)$ & $I I(\mathrm{LA}), I I I(\mathrm{LA})$ & $\bar{m}_{L L}^{I V}$ \\
\hline \hline
\end{tabular}

Table 1: The pairings of $m_{L L}$ and $m_{E}$ that can accept independent perturbations, and which have either a single massive Majorana neutrino or three degenerate neutrinos. The matrices $I, I I, I I I$, and $\bar{m}_{L L}^{I, I I, I I I, I V}$ are as defined in equations (21) and (2); the meanings of $\mathrm{U}$ and LA are described above equation (26). $A$ and $B$ are independent complex parameters with comparable magnitudes. This list is complete, up to the freedom to relabel states in the flavor basis. 


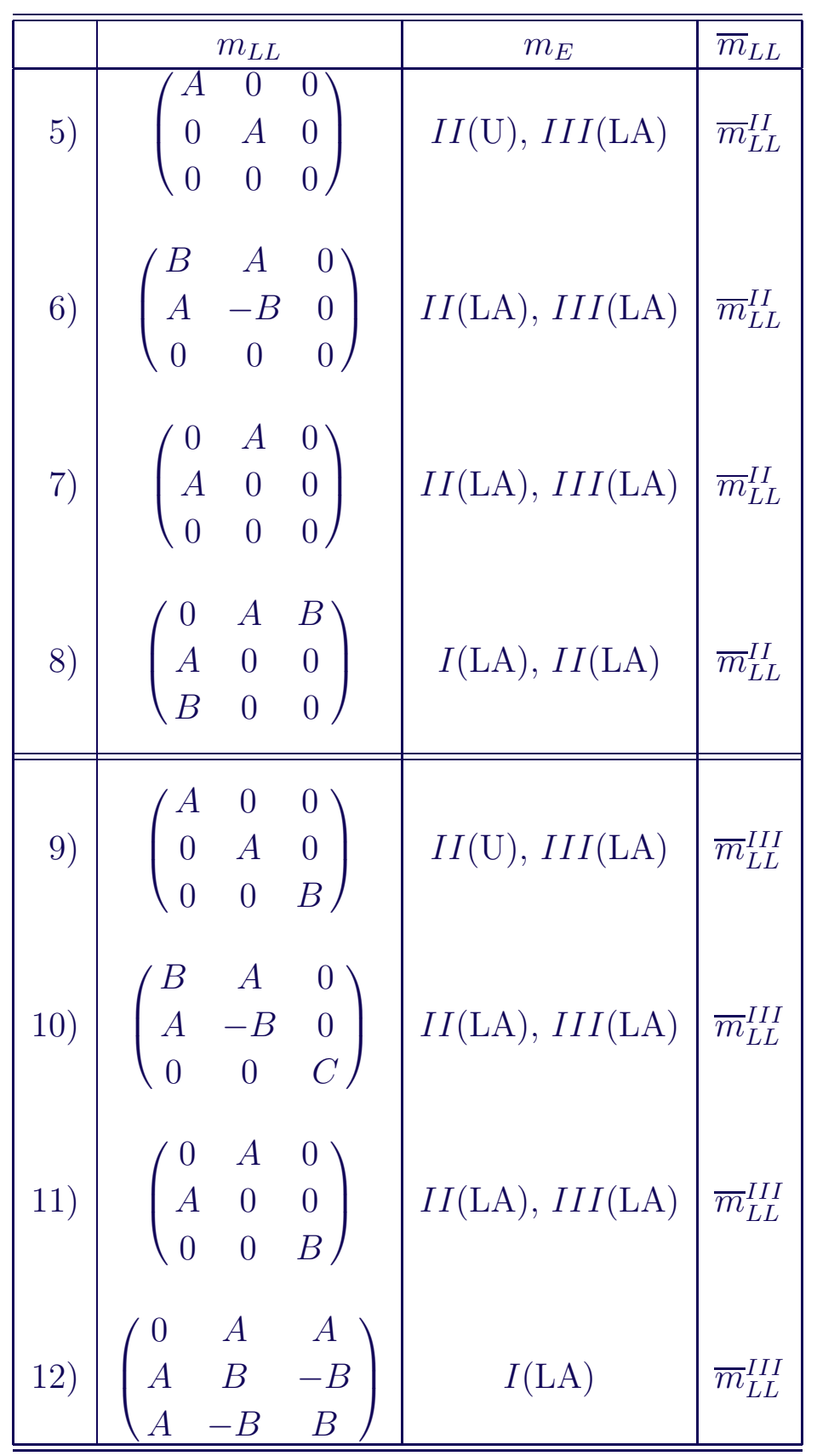

Table 2: Same as Table [1, but for $m_{L L}$ 's that have $\nu_{1}$ and $\nu_{2}$ forming a pseudo-Dirac state. $A, B$, and $C$ are again independent complex parameters, except that in cases 6 ) and 10) there are certain phase relations. 


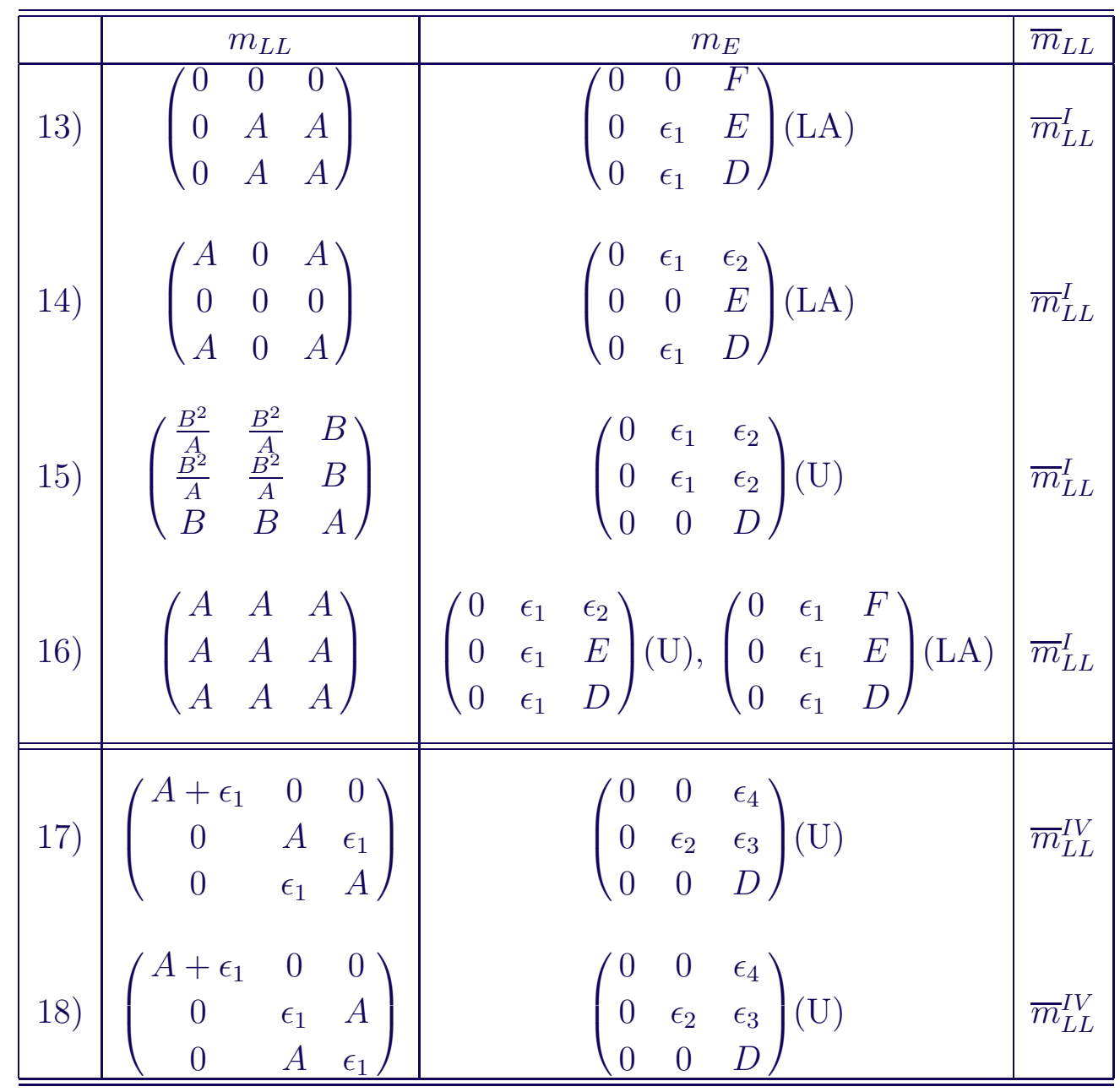

Table 3: The pairings of $m_{L L}$ and $m_{E}$ that require exact relations among perturbations, and which have either a single massive Majorana neutrino or three degenerate neutrinos. $A-F$ are independent complex parameters with comparable magnitudes, as are the perturbations $\epsilon_{1}, \epsilon_{2}, \epsilon_{3}$, and $\epsilon_{4}$. This is a complete list of leading order textures, up to the freedom to relabel states in the flavor basis. The perturbations are shown simply to illustrate, for each leading order pairing, how they can be included in a way consistent with our requirements. 


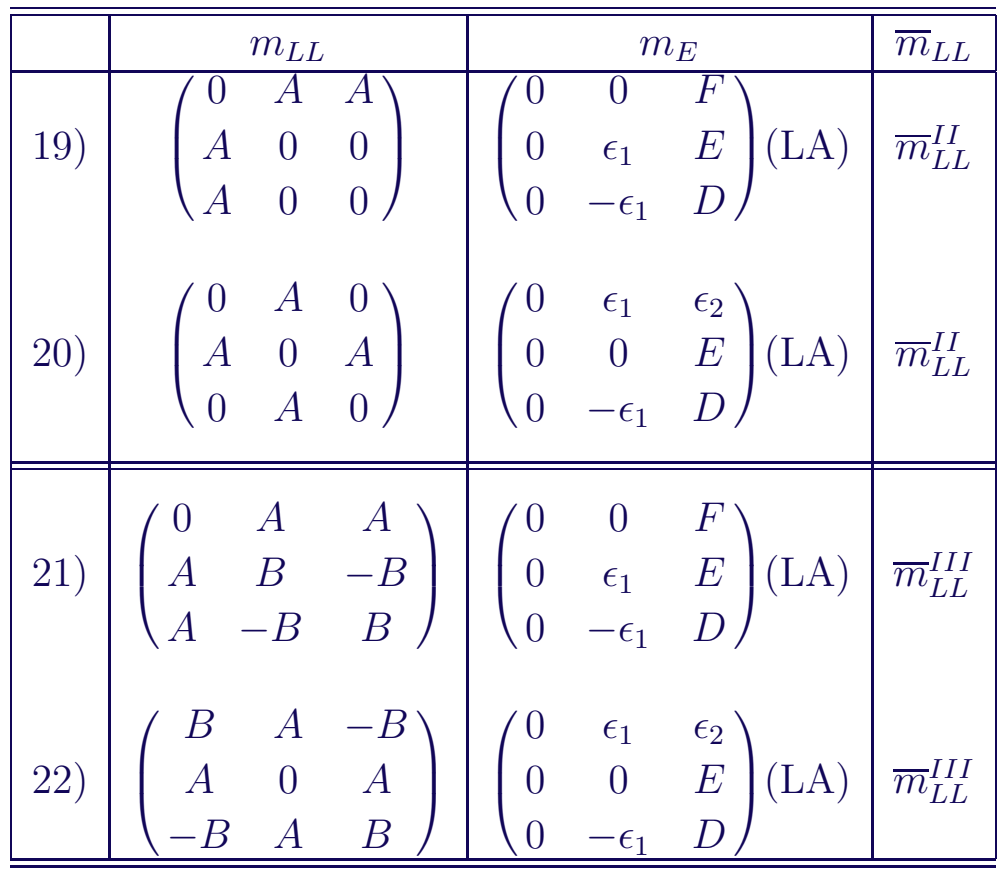

Table 4: Same as Table 3, but for $m_{L L}$ 's that have $\nu_{1}$ and $\nu_{2}$ forming a pseudo-Dirac state.

Some of the pairings of leading order $m_{L L}$ and $m_{E}$ in these tables lead to equivalent physics; for instance, the masses

$$
m_{L L}=\left(\begin{array}{ccc}
0 & 0 & 0 \\
0 & 0 & 0 \\
0 & 0 & A
\end{array}\right) \quad m_{E}=\left(\begin{array}{ccc}
0 & 0 & 0 \\
0 & 0 & E \\
0 & 0 & D
\end{array}\right)
$$

are related by a simultaneous (2-3) rotation on both the charged leptons and neutrinos to the combination

$$
m_{L L}=\left(\begin{array}{ccc}
0 & 0 & 0 \\
0 & \frac{B^{2}}{A} & B \\
0 & B & A
\end{array}\right) \quad m_{E}=\left(\begin{array}{ccc}
0 & 0 & 0 \\
0 & 0 & E^{\prime} \\
0 & 0 & D^{\prime}
\end{array}\right) .
$$

As a consequence (22) and (23) give the same form for the leptonic mixing matrix and are thus physically indistinguishable. For our purposes, (22) and (23) represent distinct cases because theories that predict the mass matrices of (22) in the flavor basis will be different from those that predict the mass matrices of (23). In other words, the apparent redundancy among some of the pairings of Tables $1-4$ arises because our rules were implemented with model-building purposes in mind.

In fact, some of the leading order $\left(m_{L L}, m_{E}\right)$ combinations that at first sight seem to lead to the same physics emerge as less similar once we consider the effects of perturbations. For example, due to the degeneracy of $\nu_{1}$ and $\nu_{2}$, we can find a simultaneous 
transformation that brings the matrices

$$
m_{L L}=\left(\begin{array}{ccc}
0 & 0 & 0 \\
0 & 0 & 0 \\
0 & 0 & A
\end{array}\right) \quad m_{E}=\left(\begin{array}{ccc}
0 & 0 & F \\
0 & 0 & E \\
0 & 0 & D
\end{array}\right)
$$

into the forms

$$
m_{L L}=\left(\begin{array}{ccc}
0 & 0 & 0 \\
0 & 0 & 0 \\
0 & 0 & A
\end{array}\right) \quad m_{E}=\left(\begin{array}{ccc}
0 & 0 & 0 \\
0 & 0 & E \\
0 & 0 & D
\end{array}\right)
$$

However, we know that this degeneracy is lifted by perturbations in $m_{L L}$, so that the similarity between (24) and (25) is somewhat artificial. For the matrices in (25), the perturbations alone determine $\theta_{12}$, which can turn out to be arbitrarily large or small. For the matrices in (24), on the other hand, we generally expect $\theta_{12} \sim 1$, barring an unlikely near-cancellation between the (1-2) rotation induced by the perturbations in $m_{L L}$ and the (1-2) rotation required to diagonalize $m_{E}$ at leading order. Note that conversely, the physical equivalence we identified between (22) and (23) does not rely on the degeneracy between $\nu_{1}$ and $\nu_{2}$, so that these matrices are on similar footing with regard to their response to perturbations.

For each pairing in Tables 1 - 4 we have identified whether, as in (25), the size of $\theta_{12}$ is fixed entirely by perturbations, so that no indication is given regarding which solutions to the solar neutrino problem are favored (denoted by "U"), or whether, as in (24), we typically have $\theta_{12} \sim 1$, so that large angle solutions are favored (denoted by "LA"). Although we have not listed them explicitly, there are in fact pairings of leading order $m_{L L}$ and $m_{E}$ that require small angle MSW solutions to the solar neutrino problem [11. For example, the pairing

$$
m_{L L}=\left(\begin{array}{ccc}
0 & A & 0 \\
A & 0 & 0 \\
0 & 0 & 0
\end{array}\right) \quad m_{E}=\left(\begin{array}{ccc}
0 & 0 & E \\
0 & 0 & E \\
0 & 0 & D
\end{array}\right)
$$

is a special case of one of the combinations in 7) from Table 2ף, and can only give a small angle MSW solution.

Up to this point we have said nothing about complex phases in our matrices. To ensure that the leading order relation $\Delta m_{12}^{2}=0$ holds, we must require that in the $m_{L L}$ 's

\footnotetext{
${ }^{5}$ We regard mass matrices obtained by setting, for example, $A=B$ in matrices from Tables 1 - 4 as special cases, and do not list them independently, even though matrices like $m_{E}=\left(\begin{array}{ccc}0 & 0 & 0 \\ 0 & 0 & E \\ 0 & 0 & D\end{array}\right)$ and $m_{E}=\left(\begin{array}{ccc}0 & 0 & 0 \\ 0 & 0 & D \\ 0 & 0 & D\end{array}\right)$ are quite different from a model builder's perspective, since different symmetries would be required to motivate them.
} 
of pairings 6 ) and 10), the $A$ 's and $B$ 's share the same phase, up to the freedom to send $\nu_{i} \rightarrow e^{i \alpha_{i}} \nu_{i}$. This means, for instance, that the $m_{L L}$ in 6) actually stands for

$$
m_{L L}=\left(\begin{array}{ccc}
B e^{2 i \alpha} & A e^{i(\alpha+\beta)} & 0 \\
A e^{i(\alpha+\beta)} & -B e^{2 i \beta} & 0 \\
0 & 0 & 0
\end{array}\right)
$$

with $\alpha$ and $\beta$ arbitrary and $A$ and $B$ real. In all other pairings, the phases of $A-F$ and the various $\epsilon$ 's are independent?.

\section{Some Special Textures}

In this section we discuss specific features of some of the more interesting pairings in Tables $1-4$.

$\theta_{23}=\frac{\pi}{4}$

One simple possibility, consistent with data from Super-Kamiokande, is that the leading order lepton masses give precisely $\theta_{23}=\frac{\pi}{4}$. For a neutrino mass matrix that requires no (2-3) rotation, the charged lepton mass matrix

$$
m_{E}=\left(\begin{array}{ccc}
0 & 0 & 0 \\
0 & 0 & D \\
0 & 0 & D
\end{array}\right)
$$

gives maximal mixing. Conversely, if the charge lepton mass matrix assumes the form

$$
m_{E}=\left(\begin{array}{ccc}
0 & 0 & 0 \\
0 & 0 & 0 \\
0 & 0 & D
\end{array}\right)
$$

then the forms for $m_{L L}$ from Tables 1 and 2 that give $\theta_{23}=\frac{\pi}{4}$ are

$$
m_{L L}=\left(\begin{array}{ccc}
0 & A & A \\
A & 0 & 0 \\
A & 0 & 0
\end{array}\right), \quad m_{L L}=\left(\begin{array}{ccc}
0 & 0 & 0 \\
0 & A & -A \\
0 & -A & A
\end{array}\right), \text { and } m_{L L}=\left(\begin{array}{ccc}
0 & A & A \\
A & B & -B \\
A & -B & B
\end{array}\right)
$$

Other pairings that give maximal mixing require exact relations among perturbations, and can be found in 17) and 18) of Table 3.

Neutrinos as hot dark matter

If there exist three light neutrinos whose splittings obey $\Delta m_{12}^{2} \ll \Delta m_{23}^{2} \sim 10^{-3} \mathrm{eV}^{2}$, then for neutrino masses to be cosmologically significant requires a high degree of degeneracy.

\footnotetext{
${ }^{6}$ Moreover, the freedom to send $\nu_{i} \rightarrow e^{i \alpha_{i}} \nu_{i}$ allows the two $A$ 's in the $m_{L L}$ of 5 ), for instance, to have different phases.
} 
Furthermore, there is a bound from neutrinoless double $\beta$ decay experiments that, in the basis where the charged lepton masses are diagonal, $m_{L L e e}<.5 \mathrm{eV}$ [12]. Lowestorder mass matrices that give degenerate neutrinos and $m_{L L e e}=0$ are thus of special interest, as they evade this experimental constraint and allow the neutrino mass scale to be cosmologically relevant. We find two combinations of $m_{L L}$ and $m_{E}$ that satisfy these criteria:

$$
m_{L L}=\left(\begin{array}{ccc}
A & 0 & 0 \\
0 & A & 0 \\
0 & 0 & A
\end{array}\right) \quad m_{E}=\left(\begin{array}{ccc}
0 & 0 & i E \\
0 & 0 & E \\
0 & \epsilon & D
\end{array}\right)
$$

and

$$
m_{L L}=\left(\begin{array}{ccc}
0 & A & 0 \\
A & 0 & 0 \\
0 & 0 & A
\end{array}\right) \quad m_{E}=\left(\begin{array}{ccc}
0 & 0 & 0 \\
0 & \epsilon_{2} & E \\
0 & \epsilon_{1} & D
\end{array}\right) .
$$

We include perturbations in $m_{E}$ because without them, the element $m_{L L e e}$ is not defined. Zeroth order lepton mass matrices

We call the contributions to $m_{L L}$ and $m_{E}$ that survive in the limit of unbroken flavor symmetry "zeroth order" masses. Consider the case of an abelian flavor symmetry, and suppose that both $m_{L L}$ and $m_{E}$ have non-vanishing elements at zeroth order. If in addition the zeroth order form of $m_{E}$ in the flavor basis is invariant under $\nu_{i} \leftrightarrow \nu_{j}$, then it follows that $\nu_{i}$ and $\nu_{j}$ are not distinguished by the flavor symmetry: if $\nu_{i} \nu_{i} h_{u}$ is an allowed operator, then so are $\nu_{i} \nu_{j} h_{u}$ and $\nu_{j} \nu_{j} h_{u}$. As a consequence it must be true that $m_{L L}$ as well is invariant under $\nu_{i} \leftrightarrow \nu_{j}$, and moreover that the (i-j) space of $m_{L L}$ must have either all entries zero, or all entries non-zero. Following this reasoning, we find that, for abelian flavor symmetries, the only pairings from Tables 1 - 4 that are candidate zeroth order mass matrices are

$$
m_{L L}=\left(\begin{array}{ccc}
0 & A & B \\
A & 0 & 0 \\
B & 0 & 0
\end{array}\right) \quad m_{E}=\left(\begin{array}{ccc}
0 & 0 & 0 \\
0 & 0 & E \\
0 & 0 & D
\end{array}\right),
$$

and

$$
m_{L L}=\left(\begin{array}{ccc}
0 & 0 & 0 \\
0 & \frac{B^{2}}{A} & B \\
0 & B & A
\end{array}\right) \quad m_{E}=\left(\begin{array}{ccc}
0 & 0 & 0 \\
0 & 0 & E \\
0 & 0 & D
\end{array}\right) .
$$

Simple seesaw-based models for the combinations in (33) and (34) were described in [9]; several other models have been based on the $m_{L L}$ of (34) [13].

\section{Democratic mass matrices}

The pairing

$$
m_{L L}=\left(\begin{array}{ccc}
0 & 0 & 0 \\
0 & 0 & 0 \\
0 & 0 & A
\end{array}\right) \quad m_{E}=\left(\begin{array}{ccc}
0 & 0 & D \\
0 & 0 & D \\
0 & 0 & D
\end{array}\right)
$$


which is a special case $(D=E=F)$ of one of the combinations in 1), results from a leading order democratic form for the charged matrix [14. A democratic form for the neutrino mass matrix is generically excluded, since it gives too large a value for $\theta_{13}$. However, it is allowed with special perturbations, as shown in pairing 16).

\section{$5 \quad$ Limitations of Texture Analysis}

Some of the requirements imposed on $m_{L L}$ and $m_{E}$ in section 2 were motivated by a desire to concentrate on mass matrices that could result most easily from flavor symmetries. One may wonder what we have missed in this regard - are there forms for $m_{L L}$ and $m_{E}$ that violate our rules but that nevertheless are plausable as consequences of flavor symmetry?

One reason that examples of such matrices can in fact be found is that if $m_{L L}$ arises by the seesaw mechanism [15], then our rules should really be applied to $m_{R R}$ and $m_{L R}$, and $m_{L L}$ should be derived from these matrices according to

$$
m_{L L}=m_{L R} m_{R R}^{-1} m_{L R}^{T}
$$

For example, the matrices

$$
m_{R R}=\left(\begin{array}{ccc}
0 & -A & A \\
-A & A & 0 \\
A & 0 & 0
\end{array}\right) \quad \text { and } \quad m_{L R}=\left(\begin{array}{ccc}
0 & 0 & A \\
0 & A & A \\
A & A & A
\end{array}\right)
$$

are certainly consistent with our rules, while the resulting mass matrix for the light neutrinos,

$$
m_{L L}=A\left(\begin{array}{lll}
1 & 2 & 3 \\
2 & 4 & 5 \\
3 & 5 & 6
\end{array}\right)
$$

clearly is not. Examples like this are not difficult to find, but it does seem to be true that in most simple cases, if $m_{R R}$ and $m_{L R}$ satisfy our rules, then so does $m_{L L}$.

Another limitation of our approach is that our rules prohibit matrix elements from being related by factors of $\frac{1}{2}, \frac{1}{3}$, etc., that could arise from Clebsch coefficients associated with the flavor group. For example, in the basis where the charged lepton masses are diagonal, the form

$$
m_{L L}=A\left(\begin{array}{ccc}
0 & \frac{1}{\sqrt{2}} & \frac{1}{\sqrt{2}} \\
\frac{1}{\sqrt{2}} & \frac{1}{2} & -\frac{1}{2} \\
\frac{1}{\sqrt{2}} & -\frac{1}{2} & \frac{1}{2}
\end{array}\right)
$$

allows (for large enough $A$ ) neutrinos to compose a significant fraction of the dark matter in the universe without violating double $\beta$ decay constraints 16. This texture corresponds to the pairing 12) of Table 2 , with $B$ chosen to be $\frac{A}{\sqrt{2}}$, in violation of our rules. On the other hand, the procedure we have used has given us a pairing of $m_{L L}$ and $m_{E}$ that leads 
precisely to the same physics as does (39). In particular, (32) for the special case $D=E$ is identical to (39) as far as the physics is concerned, and we expect that these forms may be easier to motivate with flavor symmetries.

\section{Conclusions}

The Super-Kamiokande data on atmospheric neutrino fluxes suggests that the leadingorder fermion mass matrices may not have the conventional form of (1), at least in the lepton sector. What leading order forms for lepton mass matrices are suggested by atmospheric and solar neutrino oscillations? We have derived the complete set of leading order $\left(m_{L L}, m_{E}\right)$ pairings consistent with $\Delta m_{12}^{2} \ll \Delta m_{23}^{2}, \theta_{13} \sim 0$, and $\theta_{23} \sim 1$, subject to a simplicity requirement: non-zero entries of a matrix may be equal up to a phase, or may have a precise relationship which leads to a vanishing determinant, otherwise they are independent. This simplicity requirement, motivated by an interest in textures that follow most easily from flavor symmetries, reduces an infinitely large class of matrices to the $\left(m_{L L}, m_{E}\right)$ combinations listed in Tables $1-4$.

These combinations are divided into twelve generic cases and ten special cases, according to whether the perturbations involve exact relations. For the twelve generic cases we also give the possible forms for the perurbations responsible for the muon mass. The diverse pairings we have derived lead to a variety of physics. Some give degenerate neutrinos and thus leave considerable freedom in the overall mass scale, while others, with hierarchical masses, fix the mass scale of the heaviest neutrino at $\sim 3 \times 10^{-2} \mathrm{eV}$, according to Super-Kamiokande results. The various pairings also give different predictions for $\theta_{12}$, and hence require different resolutions to the solar neutrino problem. Certainly each of our mass matrices is incomplete, because only by specifying all perturbations can the physics be fully established, yet in our view the approach we have taken offers a simple starting point for considering what mass matrices to aim for in constructing realistic theories of lepton masses.

\section{References}

[1] Super-Kamiokande Collaboration, hep-ex/9807003.

[2] G.K. Leontaris, S. Lola, and G. Ross, Nucl. Phys. B454 25 (1995).

K.S. Babu, J.C. Pati, and F. Wilczek, Link between Neutrino Masses, SuperKamiokande Result and Proton Decay, to appear.

[3] J.T. Peltoniemi, D. Tommasini, and J.W.F. Valle, Phys. Lett. B298 (1993) 383.

Q.Y. Liu and A. Yu. Smirnov, hep-ph/9712493; 
A.S. Joshipua and A. Yu. Smirnov, hep-ph/9806376;

V. Barger, S. Pakvasa, T.J. Weiler, and K. Whisnant, hep-ph/9806328;

L. Hall and N. Weiner, hep-ph/9811299.

[4] R. Barbieri, L.J. Hall, D. Smith, A. Strumia, and N. Weiner, hep-ph/9807235.

[5] X. Shi and G Fuller, astro-ph/9810075, and references cited therein.

[6] G. Altarelli and F. Feruglio, hep-ph/9809596.

[7] M. Apollonio et al., Phys. Lett. B420 (1998) 397.

[8] V.Barger, T.J. Weiler, and K. Whisnant, hep-ph/9807319;

G.L. Fogli, E. Lisi, A. Marrone, and G. Scioscia, hep-ph/9808205.

[9] R. Barbieri, L.J. Hall, and A. Strumia, hep-ph/9808333.

[10] G. Altarelli and F. Feruglio, hep-ph/9807353.

[11] L. Wolfenstein, Phys. Rev. D17 (1978) 2369;

S.P. Mikheyev and A. Yu. Smirnov, Nuovo Cimento C9 (1986).

[12] L. Baudis et al., Phys. Lett. B407 (1997) 219.

[13] Z. Berezhiani and A. Rossi, Phys. Lett. B367 (1996) 219;

M. Drees, S. Pakvasa, X. Tata, T. ter Veldhuis, Phys. Rev. D57 (1998) 5335;

S. King, hep-ph/9806440.

[14] H. Harari, H. Haut and J. Weyers, Phys. Lett. 78B (1978) 459;

Y. Koide, Phys. Rev. D28 (1983) 252; 39 (1989) 1391;

H. Fritzsch and Z. Xing, Phys. Lett. B372 (1996) 265;

M. Fukugita, M. Tanimoto and T. Yanagida, Phys. Rev. D57 (1998) 4429;

K. Kang and S. Kang, hep-ph/9802328;

M. Tanimoto, hep-ph/9807283.

[15] M. Gell-Mann, P. Ramond, R. Slansky, in Proc. it Supergravity, ed. P. van Nieuwenhuizen, D. Freedman, North Holland, 1979, p.315;

T. Yanagida, Prog. Theo. Phys. 64 (1980) 1103.

[16] F. Vissani, hep-ph/9708483;

H. Georgi and S. Glashow, hep-ph/9808293. 